\section{SPONTANEOUS RUPTURE OF HYDROCELE.}

Ed. Journal of the American Medical Association:

Dear Sir,-Tom K. called upon me with quite a large bi-lateral hydrocele-equally as large as any that $I$ had ever seen. I removed the fluid from one side in the usual manner, afterward using the compound tinct. of iodine, which restored the condition of the sac to its normal calibre. I had intended to operate upon the other during the following week, but Tom failed to come to my office until after the accident, which happened while at work in one of our theatres. He says that he heard the gurgling splash, at the same time noticing an immediate collapse of the scrotal sac, yet no flow of fluid externally visible. He suffered from considerable soreness for several days subsequent to the rupture, and upon his reporting to me I found the condition of that scrotal sac undergoing the constitutional phenomena identically as if it had been punctured, and iodine had been injected in to the sac.

No inconvenience to the patient has ever developed since the spontaneous rupture, and he is at his former occupation (an engineer).

Very respectfully,

George N. Monette, M.D.

New Orleans, May i4th, 1885 .

\section{MISCELLANEOUS.}

The Michigan State Medical Society will hold its Twentieth Annual Meeting at Port Huron, commencing Wednesday, at ro A.M., June Ioth, I885. The members of the Society, and all other physicians in Michigan who can comply with the requirements of the Society, are cordially invited to attend this meeting and identify themselves with its interests.

The Ohio State Medical Society will hold its Fortieth Annual Meeting at Dayton, June $3^{\text {rd, }} 4^{\text {th }}$, and 5 th, 1885 . The meeting will be called to order on Wednesday, June 3 rd, at 2 P.M. A good programme of work is promised, and a full attendance is expected.

New York State Medical Association, Third District Branch, will hold its First Annual Meeting at Elmira, N. Y., on Wednesday, June roth, 1885. An interesting list of papers is on the programme, and the meeting will be one of importance.

Professor P. L. Panum, of Copenhagen, who presided over the recent International Medical Congress in that city, died suddenly, May and, I 885. An interesting biographical sketch of Professor Panum was given in this journal only a few months since, in which it was announced that he would visit this country in 1887 . His death will be much regretted on both sides of the Atlantic. He had attained the age of 64 years, and was highly esteemed by all who knew him.
The Most Sensitive and Credible Tests for AlbuMEN.-At the close of an article on this subject, Dr. Henry B. Millard, of New York, draws the following conclusions: That nitric acid shows I part of albumen in 100,000. Heat shows I part in 100,000 , but rather more clearly than nitric acid, and in examinations of urine I of ten find it to show minute quantities of albumen where nitric acid does not. Tanret's test and my own test will show $\mathrm{x}$ part in 300,000 - the latter test the more clearly; this precipitates fewer of the alkaloids than Tanret's.

Nitric acid and heat show almost exactly the same reaction and percentage with artificial albumen and albuminous urine. Tanret's test and my own show the reaction better in the urine than in the artificial preparation. I think, for practical purposes and ordinary clinical use, we may show with ease, by nitric acid, I part in 100,000; heat, I part in 100,000; Tanret's test, $x$ part in 200,000; the phenic-acetic acid and potash test, $I$ part in 200,000; heat showing it more clearly than nitric acid, consequently being more sensitive, and my own test showing it more clearly than Tanret's.

Heat, although somewhat more sensitive than nitric acid, is often quite unreliable from the turbidity produced by it with mucin, and this particularly after acetic acid has been added.

Finally, there are cases in which no single reagent is sufficient, and in which in order to determine the presence of albumen, the employment of several is indispensable.-Medical Record, April 4,1885 .

The Philadelphia Polyclinic.-Dr. B. F. Baer, president of the Obstetrical Society of Philadelphia, and late demonstrator of diseases of women in the University of Pennsylvania, has been elected professor of diseases of women in the Polyclinic. Dr. H. Augustus Wilson has been elected professor of fractures and dislocations in the same institution.

RaChitis AND RHACITIS.-After an examination of the etymology of the proper term for rickets, the New York Medical Journal concludes that the word should be spelled rhachitis; that the dropping of the " $\mathrm{h}$ " is only justified by laziness.

Measles in Brooklyn.-It is stated that there have been $I, 700$ cases of measles in Brooklyn since January $\mathbf{I}$, and that seventy deaths have occurred from it.

A French Edition of Lusk's Midwifery.M. Doléris has recently completed a French translation of this valuable work, and a copy of it has been presented to the Académie de Médicine on his behalf by M. Charpentier.

Dr. WM. H. Pancoast has announced his intention of resigning the position of professor of anatomy in the Jefferson Medical College. 